\title{
Pitolisant to Treat Excessive Daytime Sleepiness and Cataplexy in Adults with Narcolepsy: Rationale and Clinical Utility
}

This article was published in the following Dove Press journal: Nature and Science of Sleep

\section{Jay T Guevarra (iD \\ Robert Hiensch (D) \\ Andrew W Varga (D) \\ David M Rapoport (ID}

Division of Pulmonary, Critical Care, and Sleep Medicine, Mount Sinai Integrative Sleep Center, Icahn School of Medicine at Mount Sinai, New York, NY I0029, USA

\begin{abstract}
Narcolepsy is a sleep disorder marked by chronic, debilitating excessive daytime sleepiness and can be associated with cataplexy, sleep paralysis and sleep-related hallucinations. Pharmacological therapy for narcolepsy primarily aims to increase wakefulness and reduce cataplexy attacks. Pitolisant is a first-in-class agent utilizing histamine to improve wakefulness by acting as an antagonist/inverse agonist of the presynaptic histamine 3 receptor. This review summarizes the clinical efficacy, safety and tolerability of pitolisant in treating the symptoms of narcolepsy. Randomized and observational studies demonstrate pitolisant to be effective in treating both hypersomnolence and cataplexy while generally being well tolerated at prescribed doses. The most common adverse reactions include headache, insomnia and nausea.
\end{abstract}

Keywords: narcolepsy, pitolisant, histamine 3 receptor, excessive daytime sleepiness, cataplexy

\section{Plain Language Summary}

Narcolepsy is a rare chronic sleep disorder associated with debilitating excessive daytime sleepiness, cataplexy, sleep-related hallucinations and sleep paralysis. It affects approximately $0.02 \%-0.05 \%$ of various populations in European, USA and Asian countries. In addition to physical symptoms, patients often have a decreased quality of life, with daytime sleepiness affecting their social and work productivity. The deficiency of a brain neuropeptide, hypocretin, has been implicated as a main cause of narcolepsy. Histamine works alongside hypocretin in regulating an individual's sleep/wake cycle. Increased histamine levels are found during wakefulness and return to lower levels during sleep. Pitolisant is the first drug in its class that increases levels of histamine by targeting the histamine 3 receptor. This review goes over the clinical outcomes, safety and tolerability of using pitolisant to treat narcolepsy.

\section{Introduction}

Narcolepsy is a clinical syndrome marked by chronic, debilitating excessive daytime sleepiness (EDS) and the presence of arousal state instability where the thresholds between the various stages of sleep and wake are easily crossed. ${ }^{1,2}$ There are two types of narcolepsy, differentiated by the presence of cataplexy (recurrent brief episodes of sudden, usually symmetrical loss of muscle tone with retained consciousness precipitated by strong emotions) and cerebral spinal fluid (CSF) hypocretin-1 levels. ${ }^{3}$ Narcolepsy type 1 (often referred to as narcolepsy with
Division of Pulmonary, Critical Care, and Sleep Medicine, Mount Sinai Integrative Sleep Center, Icahn School of Medicine at Mount Sinai, One Gustave L. Levy Place, New York, NY 10029, USA

$\mathrm{Tel}+$ I 646 339-3862

Fax + I 2128765519

Email jay.guevarra@mountsinai.org 
cataplexy) is distinguished by either the presence of cataplexy or low CSF hypocretin-1 levels $(=<110 \mathrm{pg} / \mathrm{mL})$ and is estimated to have a prevalence of $14-50$ per 100,000 people and an incidence of 4.87 per 100,000 personyears. ${ }^{4-6}$ Narcolepsy type 2 has absent cataplexy and either normal $(>110 \mathrm{pg} / \mathrm{mL})$ or unmeasured CSF hypocretin-1 levels. ${ }^{2}$ The prevalence of narcolepsy type 2 is not well studied, but estimated to be about 20-65 per 100,000 people. ${ }^{6,7}$ Other symptoms common to both narcolepsy types 1 and 2 include fragmented, disrupted sleep, hypnogogic and hypnopompic hallucinations (vivid auditory or visual dreamlike perceptions at sleep onset or offset), automatic behaviors (activities performed without awareness) and sleep paralysis (temporary inability to move while falling asleep or awakening). ${ }^{4,8}$ Patients with narcolepsy may enter rapid eye movement sleep (REM) earlier ( $<90 \mathrm{~min}$ ) after nighttime sleep initiation and have sleeponset REM periods that occur during daytime naps. Other sleep abnormalities are also common, including sleepdisordered breathing, periodic limb movements of sleep, and REM sleep behavior disorder. Depression, anxiety, and obesity also are frequently comorbid. Narcoleptic patients have a reduced quality of life, as the diagnosis affects many aspects of their day-to-day functioning, including work/school performance, social relationships and mental health. The onset of narcolepsy occurs most often between ten and 25 years of age but can occur as early as 5 years or after 40 years of age. The observation of onset during late adulthood may be confounded by delays in diagnosis. ${ }^{1}$

\section{Pathophysiology of Narcolepsy}

Wakefulness is promoted by multiple interconnected brain circuits that control ascending arousal systems. These originate from multiple nuclei that coordinate the release of wake-promoting neurotransmitters including norepinephrine, serotonin, dopamine, histamine and acetylcholine. Lateral hypothalamic neurons containing hypocretin-1 and -2 , also known as orexin A and B, sustain wakefulness by activating wake-promoting neurons in other hypothalamic regions, including the tuberomammillary nucleus, and project both rostrally to the cortex, basal forebrain and thalamus and caudally to brainstem monoaminergic nuclei, most prominently the locus coeruleus. ${ }^{9}$ Hypocretins also stimulate brain areas that inhibit REM sleep. There are only 10,000-20,000 neurons that produce hypocretin, and the selective loss of these neurons due to a presumed autoimmune etiology is implicated as the main cause of EDS and cataplexy in narcolepsy type $1 .^{10,11}$ Two receptors, hertr 1 and hcrtr 2 , mediate the effects of hypocretin in mammals. ${ }^{12}$ The absence of hcrtr 2 receptors in mice results in cataplexy and disruption during wake compared to wild-type. ${ }^{13,14}$ Dogs that lack hertr2 receptors also have the canine form of narcolepsy with cataplexy and have decreased histamine and increased dopamine concentrations in the cortex and thalamus. ${ }^{15}$

The hypocretin system acts in conjunction with histamine, which also has a pivotal role in the wake-promoting pathway in the brain by regulating the light/dark circadian cycle. It increases during wakefulness and returns to lower levels during sleep. ${ }^{16}$ Histamine originates from the tuberomammillary nucleus in the posterior hypothalamus and projects to multiple brain regions from the brainstem to the cortex. There are four different histamine receptor subtypes (H1-H4). The wakefulness activity of histamine is mediated by $\mathrm{H} 1$ receptors (H1R), which is targeted by classical antihistamines resulting in a sedating effect. The presynaptic $\mathrm{H} 3$ receptor (H3R) has a unique role as an autoreceptor due to its inhibitory effect on histamine release and formation. ${ }^{17}$ While concentrated in the central nervous system (CNS), the H3R can also be found in the pulmonary, gastrointestinal and cardiovascular systems. ${ }^{18}$ It is also present on axon terminals of non-histaminergic neurons (heteroreceptors) and has an inhibitory effect on the release of other neurotransmitters, particularly norepinephrine, $\gamma$-aminobutyric acid (GABA), dopamine, serotonin, acetylcholine and glutamate. H3Rs remain constitutively active resulting in a continued inhibitory effect even in the absence of histamine. ${ }^{19}$

Both the hypocretin and histaminergic system play coinciding roles in the control of wakefulness. ${ }^{20}$ The hypocretin system innervates histamine neurons, as proven in the rodent and zebrafish model. ${ }^{21-23}$ Histaminergic neurons receive hypocretinergic input through hcrtr2 receptors. ${ }^{24,25}$ Hypocretin-1 and hypocretin-2 depolarize the histaminergic tuberomammillary neurons and increase their firing rate through action on postsynaptic receptors. Immunocytochemical studies show that histamine and hypocretin neurons are located very close to each other. ${ }^{21}$ Hypocretin neurons are also heavily innervated by histaminergic neurons. Histamine also has an essential role in the development of the hypocretin system in zebrafish larvae, which is mediated through $\mathrm{H} 1 \mathrm{R}^{23}$ In knockout mice without H1R, hypocretin concentration in the CSF was decreased compared to control. ${ }^{26}$ In narcoleptic patients with reduced hypocretin neurons, histamine 
concentration in the CSF was also lower than healthy controls. $^{27-29}$

\section{Available Narcolepsy Medications}

There are several nonpharmacologic interventions that can help manage the symptoms of narcolepsy. These include scheduled napping, good sleep hygiene, and avoidance of drugs that can worsen daytime sleepiness. ${ }^{30}$ Most patients, however, require medications to reduce sleepiness and cataplexy. The wake-promoting agents modafinil and its R-enantiomer armodafinil are first-line medications for the treatment of EDS in narcolepsy. Their exact mechanism of action in promoting wakefulness is not fully understood but appears to be mediated by increased dopaminergic signaling by blocking dopamine reuptake. ${ }^{31}$ Stimulants, including amphetamines and methylphenidate, are secondline agents due to abuse potential and sympathomimetic side effects. Methylphenidate also inhibits dopamine and norepinephrine reuptake in the CNS while amphetamines increase both the release of dopamine and norepinephrine in the CNS and block their reuptake. Solriamfetol, a novel dopamine and norepinephrine reuptake inhibitor, was recently approved by the United States Food and Drug Administration (FDA) as a first-line treatment for adult patients with EDS in the setting of narcolepsy. ${ }^{32}$ The only drug approved by the FDA for the treatment of cataplexy is sodium oxybate whose mechanism is poorly understood but seems to be related to stimulation of $\mathrm{GABA}_{\mathrm{B}}$ receptors. It also improves EDS and can be considered a firstline therapy when cataplexy is coexistent and requires therapy. Off-label medications used for cataplexy include tricyclic antidepressants (TCAs), selective serotonin reuptake inhibitors, and serotonin and norepinephrine reuptake inhibitors. ${ }^{33}$ Many of the available treatments for narcolepsy either only partially treat its symptoms (ie wakepromoting agents without effect on cataplexy) or require complex dosing schedules and self-discipline (sodium oxybate). There is a need for new therapeutics that have limited side effects, abuse potential and efficacy on the predominant symptoms narcolepsy. ${ }^{34}$ Pitolisant (brand name Wakix $^{\circledR}$ ) is approved to treat narcolepsy without cataplexy in the United States and narcolepsy with cataplexy in Europe. It is a first-in-class agent to uniquely utilize the role of histamine in regulating wakefulness. This review aims to discuss the safety, efficacy, and tolerability of pitolisant in the management of narcolepsy.

\section{Pitolisant}

\section{Mechanism of Action}

The blockage of H3R has become a target in the management of disorders of hypersomnia, including narcolepsy. The H3R's participation in the tonic control of histamine release requires prospective antagonists to have potent intrinsic activity as inverse agonists in order to fully reverse its highly constitutive activity. ${ }^{17}$ Pitolisant, previously known as BF2.649 and tiprolisant, is the first non-imidazole-based H3R antagonist/inverse agonist to progress into clinical development.

Pitolisant is an N-piperidyl derivative that acts as a high-affinity competitive antagonist and an inverse agonist with an $\mathrm{EC}_{50}$ of $1.5 \mathrm{nM}$ and an inhibitor constant, $\mathrm{Ki}$, of $1 \mathrm{nM}$, consistent with high potency. It blocks the autoinhibiting activity of histamine and H3R agonists on endogenous histamine release (See Figure 1). It also increases histamine release above the basal level. At a low concentration of $100 \mathrm{~nm}$, it has no significant interaction with almost a hundred other human receptors and channels. ${ }^{35}$ It also increases acetylcholine release in the prefrontal cortex and hippocampus as proven in rats, enhancing arousal. Pitolisant preferentially increases dopamine in the prefrontal cortex but not in the striatal complex that comprises the nucleus accumbens. ${ }^{17}$ Pitolisant's selectivity of dopamine release was confirmed in rat models compared to modafinil, which increased dopamine release in the nucleus accumbens. This likely explains the lack of psychomotor activity and drug-abuse liability of pitolisant. ${ }^{36}$ Pitolisant is the first FDA-approved drug to treat hypersomnolence in narcolepsy without being scheduled as a controlled substance as studies in humans demonstrated that it was found to have an abuse profile similar to placebo and significantly lower than that of a phentermine, a mild stimulant. $^{37}$

\section{Pharmacokinetics}

Pitolisant was initially approved by the European Medicines Agency for narcolepsy at a dose range of $4.5-36 \mathrm{mg} /$ day. ${ }^{38} \mathrm{It}$ was then FDA-approved for the treatment of daytime

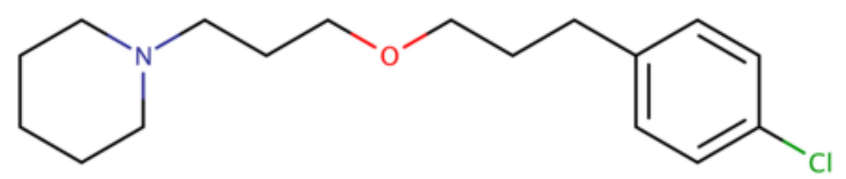

Figure I Chemical Structure of Pitolisant $\left(\mathrm{C}_{17} \mathrm{H}_{26} \mathrm{CINO}\right) .{ }^{39}$

Notes: National Center for Biotechnology Information. PubChem Database. Pitolisant, CID=9948I02, https://pubchem.ncbi.nlm.nih.gov/compound/Pitolisant (Accessed April 29, 2020) 
sleepiness in adults with narcolepsy in August 2019 at a recommended dose range of $17.8-35 \mathrm{mg} /$ day. The FDArecommended dose titration starts at $8.9 \mathrm{mg} /$ day, increasing to $17.8 \mathrm{mg} /$ day and then $35.6 \mathrm{mg} /$ day at 1 -week intervals based on tolerability. It is available in film-coated tablets, with dosage forms of $4.45 \mathrm{mg}$ and $17.8 \mathrm{mg} .{ }^{32,38}$ Pitolisant has a high affinity for H3R $\left(\mathrm{K}_{\mathrm{i}}=1 \mathrm{nM}\right)$ compared to other histamine receptors (H1R, H2R, or H4R, $\left.\mathrm{K}_{\mathrm{i}}>10 \mu \mathrm{M}\right)$. In addition, pitolisant binds to the mouse sigma-1 receptor similar to that for the H3R, which could theoretically contribute to its cognitive and mood effects. However, this interaction with the sigma-1 receptor is not entirely understood. Sigma 1-receptor agonists increase dopamine levels in the nucleus accumbens, whereas pitolisant preferentially increases its concentration in the prefrontal cortex. Pitolisant also antagonizes the sigma- 2 receptor and may diminish the effects of cocaine. ${ }^{39-41}$ Pitolisant is readily absorbed and reaches peak plasma concentration in $3 \mathrm{hrs}$. After a dose of pitolisant $35.6 \mathrm{mg}$ once daily, the steady-state $\mathrm{C}_{\max }$ is $73 \mathrm{ng}$ / $\mathrm{mL}$ and the area under the curve (AUC) is $812 \mathrm{ng} * \mathrm{hr} / \mathrm{mL}$. Pitolisant has a plasma half-life of 7.5-24.2 hrs and it reaches steady state in 5 to 6 days, and almost always by day 7 (See Table 1).

Due to its long half-life, it is recommended to take pitolisant early in the morning in order to prevent insomnia. When absorbed, pitolisant is equally distributed between red blood cells and plasma due its high serum protein binding. Metabolism of pitolisant occurs under the action of CYP3A4 and CYP2D6 and is eliminated as inactive metabolites. These metabolites are eliminated mainly through the urine $(63 \%)$, expired air $(25 \%)$ and in the feces $(<3 \%)^{38,42}$ Pitolisant dose adjustments may be needed in individuals taking medicines that impact CYP3A4 and CYP2D6.

Pitolisant dosing varies in certain populations. The plasma concentration of pitolisant is increased in patients with renal failure (stage 2-3), and the maximum daily dose recommended for these patients is $18 \mathrm{mg}$. It is not recommended for patients with end-stage renal disease. No dose change is needed for mild hepatic impairment. For patients

Table I Pitolisant Overview ${ }^{39}$

\begin{tabular}{|l|l|}
\hline $\begin{array}{l}\text { Mechanism of } \\
\text { Action }\end{array}$ & $\begin{array}{l}\text { H3 Receptor Antagonist/Inverse } \\
\text { Agonist }\end{array}$ \\
\hline Pharmacokinetics & Approximately proportional \\
$\mathrm{t}_{\max }$ median (range) & $3.5 \mathrm{~h}(2-5 \mathrm{~h})$ \\
$\mathrm{t}_{/ / 2}$ median (range) & $20 \mathrm{~h}(7.5-24.2 \mathrm{~h})$ \\
Metabolism/Clearance & CYP3A4 CYP2D6 \\
\hline
\end{tabular}

with moderate hepatic impairment (Child-Pugh B), there was a 2.4 increase in AUC and the half-life doubled. In these patients, treatment should be initiated for 2 weeks before increasing the daily dose to a similar maximum of $18 \mathrm{mg}$. Pitolisant is contraindicated in patients with severe hepatic impairment. ${ }^{42}$ Although one small study demonstrated that pitolisant at therapeutic doses does not have any inducer activity on CYP3A4, the drug's manufacturer suggests that women of reproductive potential on hormonal contraception should still use an alternative nonhormonal contraceptive during and up until 21 days after treatment due to the drug's long half-life. The effects of in utero exposure to pitolisant are unknown, but it does cross the placenta and teratogenicity was seen in some animal studies. Data collection via a pregnancy registry is ongoing to monitor pregnancy and infant outcomes following in utero exposure to pitolisant. Animal studies have demonstrated that pitolisant passes into breast milk, and its use should, therefore, be avoided in breastfeeding mothers until human studies are available. ${ }^{42}$ There are limited data on the safety and efficacy of pitolisant in elderly and pediatric populations.

CYP2D6 inhibitors such as paroxetine, fluoxetine, venlafaxine, quinidine, duloxetine, bupropion, terbinafine and cinacalcet may increase the serum concentration of pitolisant and require a $50 \%$ dose reduction of pitolisant. CYP3A4 inducers decrease levels of pitolisant and necessitate an increased dose. Antihistamines and TCAs should be avoided as they may decrease the effect of pitolisant.

\section{Cost}

Following FDA approval of pitolisant, its manufacturer Harmony Biosciences estimated an annual price tag of about $\$ 140,000$. $^{43}$ In a Swedish study, the cost per additional quality-adjusted life-year (QALY) was estimated at SEK 356,337 $(\sim € 35,600)$ and SEK 491,128 ( €49,000) for pitolisant as an adjunctive treatment. Healthcare utilization and QALYs were calculated assuming no treatment effect on survival. ${ }^{44}$

\section{Efficacy}

Lin et al first tested the histamine hypothesis in hypocretin -/- mice by enhancing its release through administering tiprolisant, a precursor of pitolisant. ${ }^{45}$ The study found tiprolisant enhanced histamine levels and noradrenergic neuron activity. When these mice were given modafinil alone, histamine cortical concentration increased by about $42 \%$ with no major difference in noradrenergic activity. In contrast, the co-administration of tiprolisant and modafinil 
resulted in a significant increase of about $216 \%$ cortical histamine concentration, as well as a $121 \%$ increase in noradrenergic activity. Clinically, tiprolisant promoted wakefulness and decreased abnormal direct onsets of REM sleep from wakefulness (DREMs), akin to a human sleep-onset REM period. Total REM sleep was decreased by tiprolisant and modafinil, but only tiprolisant considerably decreased the number and duration of DREMs episodes. The group also performed a pilot, prospective, comparative, sequential placebo-controlled, single-blind, multi-center study of a single dose of pitolisant $(40 \mathrm{mg}$ ) in 22 patients. The patients received placebo followed by tiprolisant for 1 week and were found to have a reduced Epworth Sleepiness Scale (ESS) score from baseline by 1.0 with placebo $(p>0.05)$ and 5.9 with tiprolisant $(p<$ 0.001). Patient sleep diaries suggested no differences in nocturnal sleep, including duration and wake after sleep onset number, compared to placebo.

This led to two further Phase II studies that demonstrated clinical efficacy in humans. ${ }^{46}$ Both found significant improvement in ESS score with pitolisant treatment. They also supported a titration scheme for pitolisant by starting at a lower dose and titrating upwards based on normalization of symptomatology and lack of adverse events.

\section{Pivotal Trials}

Four major Phase III studies, three of which were randomized, have evaluated the efficacy of pitolisant in patients with narcolepsy (See Table 2).

\section{Harmony I}

Harmony I was the first Phase III, randomized, doubleblind placebo-controlled trial investigating pitolisant for narcolepsy. ${ }^{47}$ It assessed the safety and efficacy of pitolisant against placebo and modafinil. Patients older than 18 years of age meeting the International Classification of Sleep Disorders 2 criteria of narcolepsy with or without cataplexy were recruited from 32 centers in five European countries. Eligible patients had a baseline ESS score of $\geq 14$. Psychostimulants were stopped for 14 or more days prior to initiation, but anti-cataplectic drugs, including sodium oxybate and antidepressants besides TCAs, were continued. Participants were randomly assigned to receive pitolisant, modafinil or placebo (1:1:1). The primary endpoint studied was a difference in change in ESS after an 8-week treatment period between pitolisant and placebo group. Secondary and additional endpoints include a variety of objective and subjective measures of sleepiness and health-related quality of life.

Patients were included if they had at least one dose of the study drug and provided at least one post-baseline value in the intention-to-treat population. Of the 94 patients that were included in the intention-to-treat analysis, 76 (81\%) had cataplexy, 42 (45\%) had previously taken psychostimulants, and 33 (35\%) were on anticataplectic drugs. Treatment lasted for 8 weeks and ranged from 10 to $40 \mathrm{mg} /$ day of pitolisant and $100-400 \mathrm{mg}$ of modafinil. For the first 3 weeks, doses were adjusted at the discretion of the investigator followed by a stable dose for the last 5 weeks. At the 8 -week result period, the pitolisant group $(-5.8 \pm 6.2)$ had a greater reduction in the ESS score compared to the placebo group $(-3.4 \pm 4.2)$, but less than the modafinil group $(-6.9 \pm 6.2)$. After adjustment for baseline differences, pitolisant was superior to placebo, but not noninferior to modafinil. When specifically looking at the responder rate (defined as an ESS $\leq 10$ at treatment conclusion), there was also a significant reduction in ESS score compared to placebo (Figure 2).

Pitolisant was also superior to placebo in most of the studied secondary efficacy endpoints, including the maintenance of wakefulness test (MWT), sustained attention to response task (SART), and modified clinical global impression of change scale (CGI-C). There was no difference between pitolisant and modafinil in these measures. A post hoc analysis demonstrated pitolisant to be superior to placebo in reduction of cataplexy rate from baseline and noninferior to modafinil as assessed through sleep diaries but no difference among treatment arms when cataplexy was assessed by the CGI-C.

In summary, Harmony I demonstrated that pitolisant reduced EDS as assessed by the ESS compared to placebo, but was not noninferior to treatment with modafinil.

\section{Harmony IBIS}

Harmony Ibis followed Harmony I with a similar study design. ${ }^{46}$ One hundred and sixty-three narcoleptic patients with $\mathrm{ESS} \geq 14$ with and without cataplexy were randomized to pitolisant, modafinil and placebo (2:2:1) over an 8-week period. Pitolisant doses were lower compared to Harmony I at 5-20 mg/day and modafinil doses ranged from 100 to $400 \mathrm{mg} /$ day. The mean ESS score reductions were $-3.6 \pm$ 5.6 in the placebo group, $-4.6 \pm 4.6$ in the pitolisant group, and $-7.8 \pm 5.9$ in the modafinil group. In contrast to Harmony I, pitolisant was not superior to placebo (difference $-1.94,95 \% \mathrm{CI}:-4.005$ to $-0.07 ; p=0.065)$ and was 


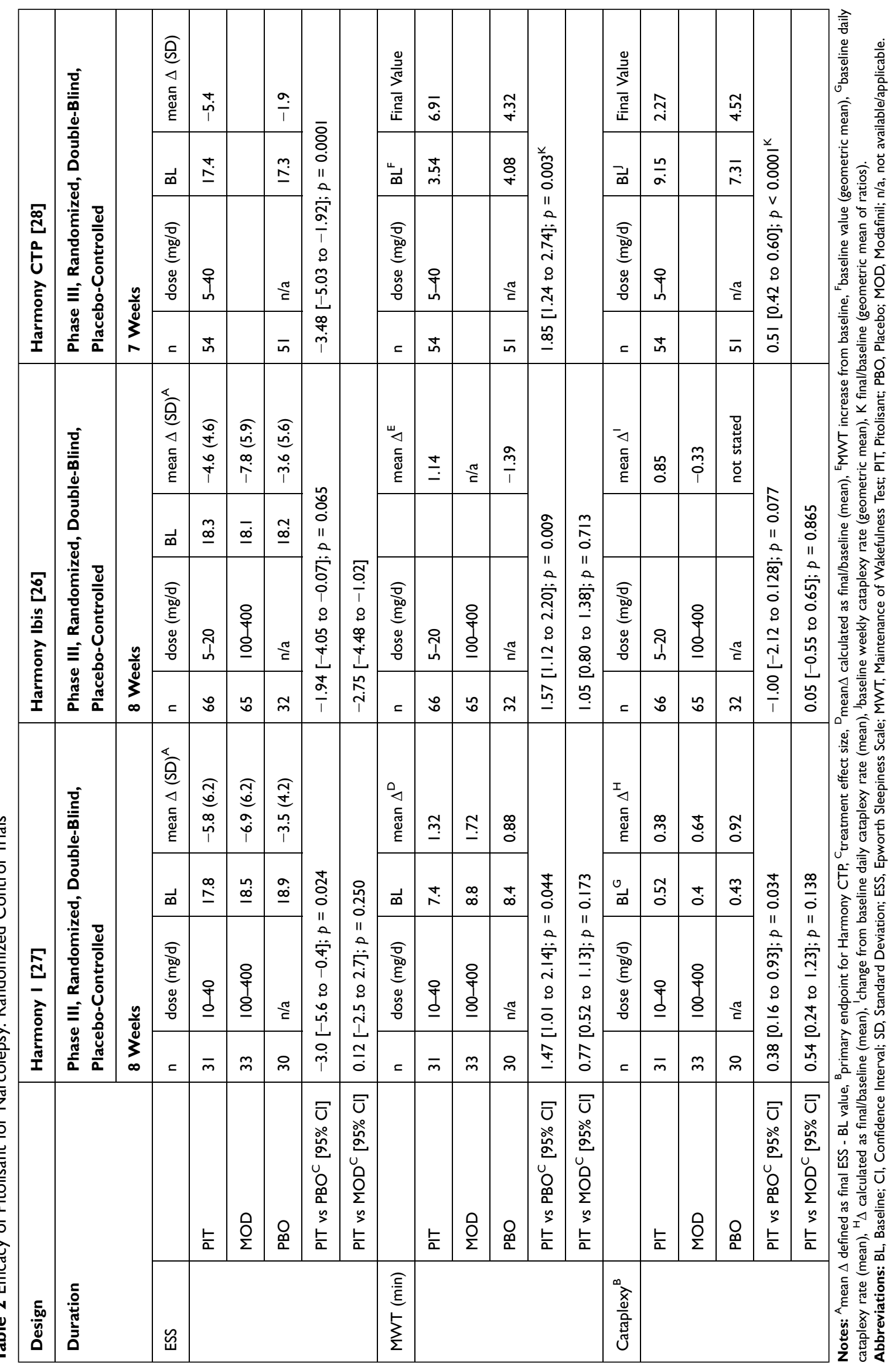




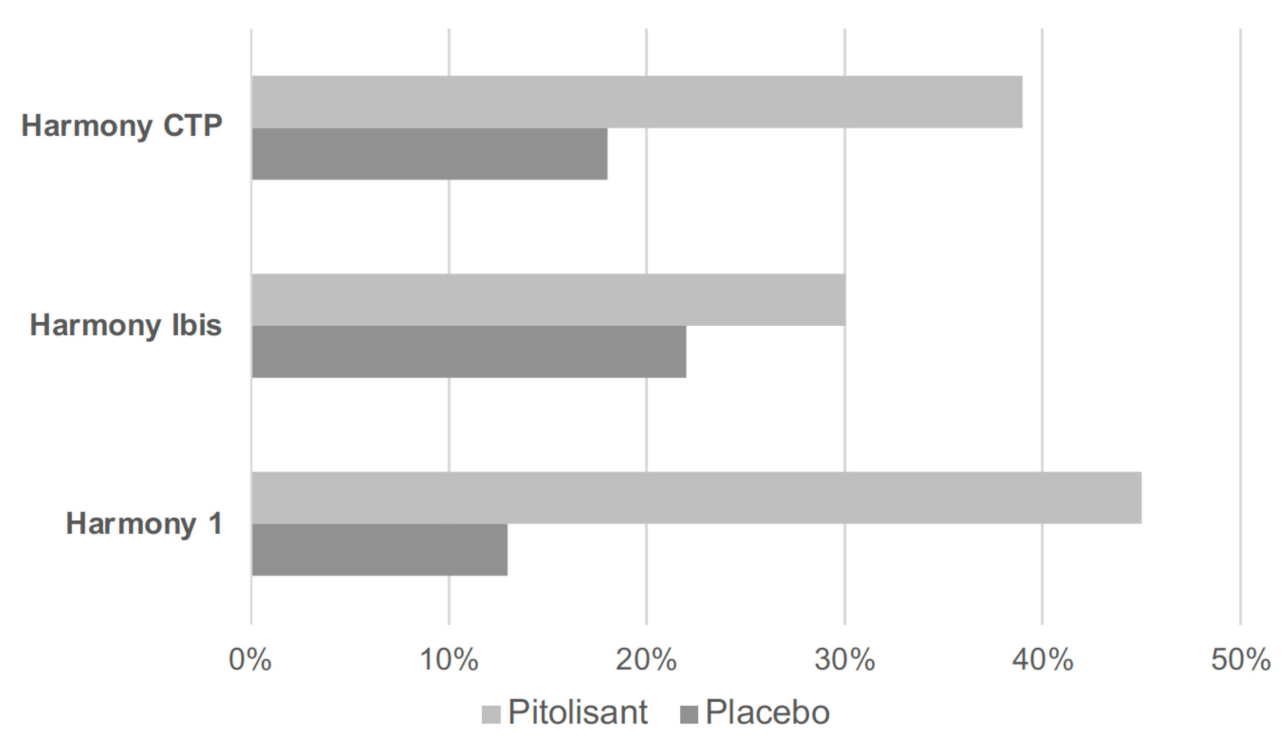

Figure 2 Epworth Sleepiness Scale Responder Rate (\%) A.47-49 $^{\text {. }}$

Notes: ${ }^{A}$ ESS $\leq 10$ or Baseline ESS - final ESS $\geq 3$ in I Harmony IBIS. ${ }^{B}$ Treatment Effect Size. $*_{p} \leq 0.05 . *^{*} p \leq 0.00$ I.

Abbreviations: ESS, Epworth Sleepiness Scale; PIT, pitolisant; PBO, placebo.

again not noninferior to modafinil (difference $-2.75,95 \%$ CI: -4.48 to $-1.02,<3$ points) for the primary outcome of improving mean ESS score. Patients receiving pitolisant did have a higher ESS responder rate score compared to placebo (OR 9.24 [95\% CI: 3.82 to 22.35]; $p=0.001$ ) and a similar rate to modafinil (OR 1.06 [0.44 to 2.54]; $p=0.894$ ) (See Figure 2). In post hoc sensitivity analysis, a superiority test demonstrated modafinil as superior to placebo in final ESS. MWT scores were significantly increased in the pitolisant group $(\Delta=+1.14 \mathrm{~min})$ with a decrease in the placebo group $(\Delta=-1.39 \mathrm{~min})$ during the final visit. Other secondary endpoints including SART, CGI-C, subjective EDS symptoms confirmed previous positive efficacy data seen in pitolisant in comparison to placebo.

Unlike Harmony I, pitolisant was not demonstrated to reduce cataplexy rate in comparison to placebo. In both studies, pitolisant, modafinil and placebo recipients did not differ significantly with respect to the quality of life from baseline.

\section{Harmony CTP}

Harmony CTP was a randomized, double-blind, placebocontrolled trial focusing on the safety and efficacy of pitolisant on cataplexy events in patients with narcolepsy with cataplexy. ${ }^{48}$ Patients with a history of at least three cataplexy episodes per week and EDS (ESS $\geq 12$ ) were recruited from 16 sleep centers in nine countries and randomly assigned to receive either pitolisant or placebo once per day. Treatment duration was 7 weeks with the first 3 weeks used for flexible dosing based on tolerance and efficacy by clinical assessment (range $5-20 \mathrm{mg}$ /day) followed by 4 weeks of stable dosing (5-40 mg/day). The primary endpoint was the change in an average number of cataplexy attacks per week (ie weekly cataplexy rate (WCR)) from baseline ( $\left.\mathrm{WCR}_{\text {baseline }}\right)$ to the WCR during 4 weeks of stable treatment $\left(\mathrm{WCR}_{\text {final }}\right)$.

A total of 54 patients were randomized into the pitolisant group and 51 to the placebo group in the intention-to-treat analysis. Pitolisant reduced the WCR compared to baseline by $75 \%\left(\mathrm{WCR}_{\text {final }}=2.27 ; \mathrm{WCR}_{\text {baseline }}=9.15 ; \mathrm{WCR}_{\text {final } / \text { baseline }}=\right.$ 0.25 ), while in the placebo group, the reduction was only $38 \%$ $\left(\mathrm{WCR}_{\text {final }}=4.52 ; \mathrm{WCR}_{\text {baseline }}=7.31 ; \mathrm{WCR}_{\text {final/baseline }}=0.62\right)$. The rate ratio was 0.512 and statistically significant in favor of pitolisant (95\% CI 0.43-0.6; $p<0.0001)$. Pitolisant was also associated with significant improvement in comparison to placebo in secondary outcomes including ESS and MWT.

\section{Harmony III}

Harmony III, a phase III, pragmatic, prospective, openlabel study was an uncontrolled trial designed to assess the long-term safety of pitolisant in the treatment of EDS (ESS $\geq 12$ ) in narcolepsy patients with or without cataplexy regardless of previous treatment. ${ }^{49}$ Of the 102 patients who received pitolisant, 29 were treatment naïve. Concomitant stimulants, H1 antagonists, and anticataplectic agents, except for TCAs, were allowed. A total of 68 patients (51 with cataplexy) completed the 12 months of treatment and had a mean decrease in ESS

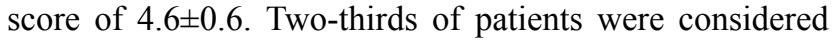


responders, defined as a final ESS $\leq 10$ or an ESS decrease $\geq 3$ points. The 1 -year response rate (final ESS score $\leq 10$ and/or decrease $\geq 3$ ) was $64.7 \%$ (44/68), and ESS score normalized $(\leq 10)$ in $36.8 \%(25 / 68)$. The largest improvement was seen in patients taking pitolisant monotherapy de novo ( $-6.5 \pm 1.3$ ), although mean ESS score was higher in these patients at baseline. In patients with generalized and partial cataplexy, cataplexy rates decreased by $88 \%$ and $71 \%$, respectively ( $p=0.055$ ). Frequency of hallucinations, sleep paralysis and sleep attacks were reduced by $54 \%, 63 \%$, and $27 \%$, respectively.

The limitations of Harmony III, including its openlabel design, lack of reference therapy, concomitant presence of drug-naïve and already-treated patients, and lack of objective measures of EDS do not allow for impartial conclusions of its efficacy. Nevertheless, it was the first pragmatic trial looking at the long-term effects of pitolisant in a realistic population and in conjunction with other therapies. It supports pitolisant as a relatively welltolerated medication for narcoleptics with or without cataplexy.

\section{Safety and Tolerability}

\section{Adverse Reactions}

The randomized, placebo-controlled Harmony trials, a USbased pitolisant expanded access clinical evaluation (PEACE) program, and supportive data from clinical trials studying the efficacy of pitolisant in other hypersomniarelated diagnoses have informed the safety profile of pitolisant (See Table 3). ${ }^{50}$ Placebo-controlled trials best identify adverse reactions directly attributable to treatment therapy. Adverse reactions that occurred at least twice the rate of placebo and were seen in $\geq 5 \%$ of patients include insomnia $(6 \%)$, nausea $(6 \%)$, and anxiety $(5 \%)$. Other adverse reactions common to both pitolisant and placebo included headache ( $18 \%$ in pitolisant vs $15 \%$ in placebo), upper respiratory tract infection ( $5 \%$ vs $3 \%$ ), musculoskeletal pain ( $5 \%$ vs $3 \%$ ), and anxiety (5\% vs 1\%). ${ }^{42}$ The Harmony III study suggested that the frequency of adverse events decreases during the course of treatment: $54 \%$ reported adverse events over the first 3 months versus $12.5 \%$ during the last 3 months. 13.1\% of adverse events in this study were considered severe but only half considered related to the study drug, and all but one patient had complete resolution of the adverse event. Patents who were taking additional anti-narcoleptic agents had a twice greater frequency of adverse events compared to patients who took pitolisant alone $(53.7 \%$ versus $29.2 \%$, $p=0.012)$. A recent abstract integrating the safety and tolerability data from four randomized, placebo-controlled trials corroborated these findings. ${ }^{51}$ Adverse events for pitolisant and placebo occurred in $49.4 \%$ and $41.2 \%$ patients, respectively. Only two in the pitolisant group were considered serious. No clinically significant effects were seen in vital signs, laboratory findings, or ECG parameters. Pitolisant was determined to be generally safe and well tolerated. The PEACE program further substantiated tolerability and safety. Despite most patients (91.1\%) taking maximal dose and many (60.1\%) taking additional narcolepsy medications, only $8.2 \%$ patients discontinued therapy$5.3 \%$ from adverse events and $1.1 \%$ for lack of efficacy. The most reported adverse events included headache (8.1\%), anxiety $(3.8 \%)$ and nausea $(3.4 \%){ }^{52}$ The incidence of adverse effects does appear to be related to dose. A large study involving over 1800 subjects taking pitolisant for a variety of etiologies (including off-label conditions) found that the incidence of adverse events was dose-dependent $(11.1 \%$ at $4.5 \mathrm{mg} / \mathrm{d} ; 20.3 \%$ at $9 \mathrm{mg} / \mathrm{d} ; 32.5 \%$ at $18 \mathrm{mg} / \mathrm{d}$; $36.4 \%$ at $36 \mathrm{mg} / \mathrm{d}$ ). Again, the most common adverse effects were neuropsychiatric, including insomnia (8.4\%), headache (7.7\%), anxiety (2.1\%), irritability (1.8\%), dizziness (1.4\%), depression $(1.3 \%)$, tremor $(1.2 \%)$, sleep disorders $(1.1 \%)$ and vertigo (1.0\%). Gastrointestinal effects including nausea (4.8\%), vomiting (1\%) and diarrhea (1\%) were also observed. $^{53}$

\section{QTc Prolongation}

The package insert for pitolisant states that it prolongs the QT interval, and it recommends that patients with known QT prolongation, taking medications that prolong the QT, or those otherwise at risk for arrhythmias avoid its use. Randomized studies that look at pitolisant prescribed at recommended doses $(40 \mathrm{mg})$, however, have not demonstrated a significant increase in the QTc in comparison to placebo. ${ }^{46}$ In contrast, various supratherapeutic doses (120-240 mg) increased the QTc by about $10 \mathrm{~ms}$. While clinical trials using therapeutic doses did not identify any cardiac safety issues, caution is advised in at-risk patients. ${ }^{46}$

\section{Nighttime Sleep}

The effect of pitolisant on nighttime sleep with narcolepsy was recently studied. ${ }^{54}$ Data collected included the subjective Pittsburgh Sleep Quality Index (PSQI) and objective overnight polysomnography (PSG). Post-treatment and baseline PSG parameters were similar including total recorded sleep time (354.1 vs $365.9 \mathrm{~min}$ ), sleep efficiency 
Table 3 Pitolisant Adverse Reactions ${ }^{\text {a50 }}$

\begin{tabular}{|c|c|c|c|}
\hline Adverse Effect & $\begin{array}{l}\text { Pitolisant (\%) } \\
(\mathrm{n}=\text { I } 52)\end{array}$ & $\begin{array}{l}\text { Placebo }(\%) \\
(n=1 \mid 4)\end{array}$ & Includes the Following Terms: \\
\hline Headache & 18 & 15 & Migraine, premenstrual, cluster, tension \\
\hline Insomnia & 6 & 2 & Initial, middle insomnia, poor quality sleep \\
\hline Nausea & 6 & 3 & \\
\hline Upper Respiratory Infection & 5 & 3 & Pharyngitis, rhinitis, sinusitis, inflammation, viral \\
\hline Musculoskeletal Pain & 5 & 3 & $\begin{array}{l}\text { arthralgia, back pain, carpal tunnel Syndrome, limb discomfort, myalgia, neck } \\
\text { pain, osteoarthritis, pain in extremity, sciatica }\end{array}$ \\
\hline Anxiety & 5 & I & Nervousness, stress, stress at work \\
\hline Increased Heart Rate & 3 & 0 & Sinus tachycardia, tachycardia \\
\hline Hallucinations & 3 & 0 & Visual, hypnagogic \\
\hline Irritability & 3 & 2 & \\
\hline Abdominal Pain & 3 & I & Abdominal discomfort, upper abdominal pain \\
\hline Sleep Disturbance & 3 & 2 & Dyssomnia, sleep disorder, sleep paralysis, sleep talking \\
\hline Decreased Appetite & 3 & 0 & \\
\hline Cataplexy & 2 & I & \\
\hline Dry Mouth & 2 & 1 & \\
\hline Rash & 2 & I & Eczema, erythema migrans, rash, urticaria \\
\hline
\end{tabular}

Note: ${ }^{a}$ Adverse reaction that occur in $\geq 2 \%$ of patients from three pooled randomized, placebo-controlled narcolepsy studies.

(79.6 vs $78.9 \%)$ and arousal index (17.2 vs 18.1 arousals/ hour). No change was observed on the overall PSQI, although the component of sleep efficiency was negatively affected.

\section{Abuse Potential}

Animal studies of pitolisant demonstrate a low potential of abuse. $^{36,55}$ This was validated in a human study in a randomized, double-blind, active- and placebo-controlled four-period crossover study. ${ }^{37}$ Nondependent recreational stimulant users able to distinguish a known drug of abuse, phentermine, from placebo in a drug discrimination test were recruited and randomized to receive pitolisant at therapeutic (35.6 $\mathrm{mg}$ ) and supratherapeutic doses $(213.6 \mathrm{mg})$, phentermine $60 \mathrm{mg}$, and placebo. Using the primary endpoint of maximum effect $\left(E_{\max }\right)$ on the 100-point Drug Liking visual analog scale, $\mathrm{E}_{\max }$ was similar between both pitolisant doses and placebo and significantly greater for phentermine, supporting a minimal abuse potential for pitolisant.

\section{Pitolisant and Other Narcolepsy Medications}

Comparative studies investigating different medications and optimal drug doses for patients with narcolepsy types 1 and 2 are sparse. A meta-analysis looked at 14 randomized controlled trials in an attempt to compare the efficacy between multiple treatments for narcolepsy using a random model that assumed heterogeneity between studies and corrected for multi-arm studies. ${ }^{56}$ Medications included sodium oxybate (6 and $9 \mathrm{~g} / \mathrm{d}$ ), modafinil (between 200 and $400 \mathrm{mg} / \mathrm{d}$ ), and pitolisant (up to 20 and up to $40 \mathrm{mg} / \mathrm{d}$ ). Although significant heterogeneity ( $>50 \%$ ) was found between 12/14 studies for almost all endpoints, between-design consistency was present. The meta-data analysis determined that pitolisant up to $40 \mathrm{mg} / \mathrm{d}$, sodium oxybate $9 \mathrm{~g} / \mathrm{d}$, and modafinil $200-400 \mathrm{mg} / \mathrm{d}$ had similar efficacy for EDS as determined by the ESS and MWT. For cataplexy, pitolisant $40 \mathrm{mg} / \mathrm{d}$ and sodium oxybate $9 \mathrm{~g} / \mathrm{d}$ had similar efficacy. Although the effects between medications on average were comparable, it remains to be determined whether patients who do not respond well to any given medicine will respond to medicines in another class or whether a medicine's effects may be potentiated by combination with a medicine from another class.

\section{Summary}

Narcolepsy is a debilitating sleep disorder marked by EDS and, in many patients, cataplexy. The pharmacological treatment options are slim but expanding. Modafinil and armodafinil have historically been considered first-line options for EDS. Sodium oxybate is the only FDAapproved medication to treat cataplexy secondary to narcolepsy and is effective in reducing sleep fragmentation. ${ }^{57}$ Pitolisant is a new addition to the pharmacological armamentarium to treat EDS in narcolepsy, with several trials 
suggesting efficacy in cataplexy as well. Pitolisant is a first-in-class wake-promoting medication that works as an antagonist/inverse agonist of the H3R and is the only narcolepsy drug not scheduled as a controlled substance. It is generally well tolerated, with headache, insomnia, nausea and anxiety being the most common adverse reactions. Large, longitudinal studies of pitolisant's effect on both EDS and cataplexy in narcolepsy as well as comparative and medication combination studies are needed to help guide the overall rationale for physicians in narcolepsy management. Pitolisant certainly will develop a niche in the treatment algorithm in patients with narcolepsy, who often remain with symptoms despite current recommended therapy.

\section{Disclosure}

Dr. Jay T. Guevarra and Dr. Robert Hiensch have no disclosures. Dr. Andrew W. Varga has previously served as a consultant for Merck and Eisai Pharmaceuticals and reports grants, personal fees, and non-financial support from Merck, and personal fees from Eisai, outside the submitted work. $\mathrm{He}$ is supported by: NIH/NIA R01AG056682, R01AG066870, R21AG059179, the Alzheimer's Association, the McClung Foundation, and the Merck Investigators Studies Program. Dr. David M. Rapoport receives patent royalties from Fisher \& Paykel Healthcare Limited and Sefam Medical, Ltd for CPAP modifications; consulting fees and grant support from Fisher \& Paykel Healthcare Limited; and consulting fees from BioMarin Pharmaceutical Inc, Morphy, Inc, and Jazz Pharmaceuticals plc. The authors report no other potential conflicts of interest for this work.

\section{References}

1. Scammell TE. Narcolepsy. N Engl J Med. 2015;373(27):2654-2662. doi:10.1056/NEJMra1500587

2. Thorpy MJ. Narcolepsy, a Clinical Guide. 2nd ed. Switzerland: Springer International Publishing; 2015.

3. Darien I. International Classification of Sleep Disorders. $3^{\text {rd }}$ ed. American Academy of Sleep Medicine;2014.

4. Longstreth WT, Koepsell TD, Ton TG, Hendrickson AF, van Belle G. The epidemiology of narcolepsy. Sleep. 2007;30(1):13-26. doi:10.1093/sleep/30.1.13

5. Hale L, Guan S, Emanuele E. Narcolepsy: A Clinical Guide. 2nd ed. Switzerland: Springer International Publishing; 2015.

6. Scheer D, Schwartz SW, Parr M, Zgibor J, Sanchez-Anguiano A, Rajaram L. Prevalence and incidence of narcolepsy in a US health care claims database, 2008-2010. Sleep. 2019;42.

7. Silber MH, Krahn LE, Olson EJ, Pankratz VS. The epidemiology of narcolepsy in Olmsted County, Minnesota: a population-based study. Sleep. 2002;25(2):197-202. doi:10.1093/sleep/25.2.197
8. Guilleminault C, Billiard M, Montplaisir J, Dement WC. Altered states of consciousness in disorders of daytime sleepiness. J Neurol Sci. 1975;26(3):377-393. doi:10.1016/0022-510X(75)90209-9

9. Scammell TE, Arrigoni E, Lipton JO. Neural circuitry of wakefulness and sleep. Neuron. 2017;93(4):747-765. doi:10.1016/j.neuron.2017. 01.014

10. Mahoney CE, Cogswell A, Koralnik IJ, Scammell TE. The neurobiological basis of narcolepsy. Nat Rev Neurosci. 2019;20(2):83-93. doi:10.1038/s41583-018-0097-x

11. Nishino S, Ripley B, Overeem S, Lammers GJ, Mignot E. Hypocretin (orexin) deficiency in human narcolepsy. Lancet. 2000;355 (9197):39-40. doi:10.1016/S0140-6736(99)05582-8

12. Sakurai T. The neural circuit of orexin (hypocretin): maintaining sleep and wakefulness. Nat Rev Neurosci. 2007;8(3):171-181. doi:10.1038/nrn2092

13. Willie JT, Chemelli RM, Sinton CM, et al. Distinct narcolepsy syndromes in Orexin receptor-2 and Orexin null mice: molecular genetic dissection of Non-REM and REM sleep regulatory processes. Neuron. 2003;38(5):715-730. doi:10.1016/S0896-6273(0 3) $00330-1$

14. Willie JT, Renthal W, Chemelli RM, et al. Modafinil more effectively induces wakefulness in orexin-null mice than in wild-type littermates. Neuroscience. 2005;130(4):983-995. doi:10.1016/j.neuroscience. 2004.10.005

15. Lin L, Faraco J, Li R, et al. The sleep disorder canine narcolepsy is caused by a mutation in the hypocretin (orexin) receptor 2 gene. Cell. 1999;98(3):365-376. doi:10.1016/S0092-8674(00)81965-0

16. Haas HL, Sergeeva OA, Selbach O. Histamine in the nervous system. Physiol Rev. 2008;88:1183-1241.

17. Schwartz JC. The histamine H3 receptor: from discovery to clinical trials with pitolisant. $B r \quad J$ Pharmacol. 2011;163(4):713-721. doi:10.1111/j.1476-5381.2011.01286.x

18. Ghamari N, Zarei O, Arias-Montaño JA, et al. Histamine H. Pharmacol Ther. 2019;200:69-84. doi:10.1016/j.pharmthera. 2019.04.007

19. Schlicker E, Kathmann M. Role of the Histamine H. Handb Exp Pharmacol. 2017;241:277-299.

20. Sundvik M, Panula P. Interactions of the orexin/hypocretin neurones and the histaminergic system. Acta Physiol (Oxf). 2015;213 (2):321-333. doi:10.1111/apha.12432

21. Eriksson KS, Sergeeva O, Brown RE, Haas HL. Orexin/hypocretin excites the histaminergic neurons of the tuberomammillary nucleus. J Neurosci. 2001;21(23):9273-9279. doi:10.1523/JNEUROSCI.2123-09273.2001

22. Kaslin J, Nystedt JM, Ostergård M, Peitsaro N, Panula P. The orexin/ hypocretin system in zebrafish is connected to the aminergic and cholinergic systems. $J$ Neurosci. 2004;24(11):2678-2689. doi:10.1523/JNEUROSCI.4908-03.2004

23. Sundvik M, Kudo H, Toivonen P, Rozov S, Chen YC, Panula P. The histaminergic system regulates wakefulness and orexin/hypocretin neuron development via histamine receptor $\mathrm{H} 1$ in zebrafish. FASEB J. 2011;25(12):4338-4347. doi:10.1096/fj.11-188268

24. Marcus JN, Aschkenasi CJ, Lee CE, et al. Differential expression of orexin receptors 1 and 2 in the rat brain. J Comp Neurol. 2001;435 (1):6-25. doi:10.1002/cne.1190

25. Yamanaka A, Tsujino N, Funahashi H, et al. Orexins activate histaminergic neurons via the orexin 2 receptor. Biochem Biophys Res Commun. 2002;290(4):1237-1245. doi:10.1006/bbrc.2001.6318

26. Lin L, Wisor J, Shiba T, et al. Measurement of hypocretin/orexin content in the mouse brain using an enzyme immunoassay: the effect of circadian time, age and genetic background. Peptides. 2002;23 (12):2203-2211. doi:10.1016/S0196-9781(02)00251-6

27. Nishino S, Sakurai E, Nevsimalova S, et al. Decreased CSF histamine in narcolepsy with and without low CSF hypocretin-1 in comparison to healthy controls. Sleep. 2009;32(2):175-180. doi:10.1093/sleep/ 32.2.175 
28. Bassetti CL, Baumann CR, Dauvilliers Y, Croyal M, Robert P, Schwartz JC. Cerebrospinal fluid histamine levels are decreased in patients with narcolepsy and excessive daytime sleepiness of other origin. J Sleep Res. 2010;19(4):620-623. doi:10.1111/j.13652869.2010.00819.x

29. Kanbayashi T, Kodama T, Kondo H, et al. CSF histamine contents in narcolepsy, idiopathic hypersomnia and obstructive sleep apnea syndrome. Sleep. 2009;32(2):181-187. doi:10.1093/sleep/32.2.181

30. Neikrug AB, Ong JC. Narcolepsy: A Clinical Guide. 2nd ed. Switzerland: Springer International Publishing; 2015.

31. Volkow ND, Fowler JS, Logan J, et al. Effects of modafinil on dopamine and dopamine transporters in the male human brain: clinical implications. JAMA. 2009;301(11):1148-1154. doi:10.1001/ jama.2009.351

32. Thorpy MJ. Recently approved and upcoming treatments for narcolepsy. CNS Drugs. 2020;34(1):9-27. doi:10.1007/s40263-01900689-1

33. Barateau L, Lopez R, Dauvilliers Y. Management of narcolepsy. Curr Treat Options Neurol. 2016;18(10):43. doi:10.1007/s11940-0160429-y

34. de Biase S, Gigli GL, Valente M. Important decisions in choosing the pharmacotherapy for narcoleptics. Expert Opin Pharmacother. 2019;20(5):483-486. doi:10.1080/14656566.2018.1561861

35. Syed YY. Pitolisant: first global approval. Drugs. 2016;76 (13):1313-1318. doi:10.1007/s40265-016-0620-1

36. Uguen M, Perrin D, Belliard S, et al. Preclinical evaluation of the abuse potential of Pitolisant, a histamine $\mathrm{H} 3$ receptor inverse agonist/ antagonist compared with Modafinil. Br J Pharmacol. 2013;169 (3):632-644. doi:10.1111/bph.12149

37. Setnik B, McDonnell M, Mills C, et al.Evaluation of the abuse potential of pitolisant, a selective H3-receptor antagonist/inverse agonist, for the treatment of adult patients with narcolepsy with or without cataplexy. Sleep. 2019.

38. Wakix:Highlights of prescribing information. Plymouth Meeting, PA. 2020

39. Riddy DM, Cook AE, Shackleford DM, et al. Drug-receptor kinetics and sigma-1 receptor affinity differentiate clinically evaluated histamine $\mathrm{H}(3)$ receptor antagonists. Neuropharmacology. 2019;144:244-255. doi:10.1016/j.neuropharm.2018.10.028

40. EMA. EMA. Wakix Assessment Report. 2015

41. Lamb YN. Pitolisant: a review in narcolepsy with or without cataplexy. CNS Drugs. 2020;34(2):207-218. doi:10.1007/s40263020-00703-x

42. Wakix. Summary of product characteristics. Bioprojet Pharma. 2016

43. Emerson E. Newly approved narcolepsy product has a price tag exceeding 100K annually. Crumdale Partners. 2020.
44. Bolin K, Niska P, Pirhonen L, Wasling P, Landtblom AM. The cost utility of pitolisant as narcolepsy treatment. Acta Neurol Scand. 2019.

45. Lin JS, Dauvilliers Y, Arnulf I, et al. An inverse agonist of the histamine $\mathrm{H}(3)$ receptor improves wakefulness in narcolepsy: studies in orexin-/- mice and patients. Neurobiol Dis. 2008;30(1):74-83. doi:10.1016/j.nbd.2007.12.003

46. EMA. Wakix assessment. 2020. Available from: https://www.ema. europ. Accessed August 18, 2020.

47. Dauvilliers Y, Bassetti C, Lammers GJ, et al. Pitolisant versus placebo or modafinil in patients with narcolepsy: a double-blind, randomised trial. Lancet Neurol. 2013;12(11):1068-1075. doi:10.1016/ S1474-4422(13)70225-4

48. Szakacs Z, Dauvilliers Y, Mikhaylov V, et al. Safety and efficacy of pitolisant on cataplexy in patients with narcolepsy: a randomised, double-blind, placebo-controlled trial. Lancet Neurol. 2017;16 (3):200-207. doi:10.1016/S1474-4422(16)30333-7

49. Dauvilliers Y, Arnulf I, Szakacs Z, et al. Long-term use of pitolisant to treat patients with narcolepsy: harmony III Study. Sleep. 2019:42.

50. Solanges M. Pitolisant: Clinical Review. Rev of Safety. Data provided by the U.S. Food and Drug Administration. 2019;332. Available from: https://www.accessdata.fda.gov. Accessed August 18, 2020

51. Scart-Grès C, Momah C, Roy M, Maski K, Piris S, RK B. Safety and tolerability of pitolisant in the treatment of adults. Sleep. 2019;42 (Supplement_1):A244-5. doi:10.1093/sleep/zsz067.612

52. Bauer E, Davis C, Patroneva A, Dayno J, Thorpy M. The safety and tolerability of pitolisant in the treatment of excessive daytime sleepiness and cataplexy in adult patients with narcolepsy: an open-label, expanded access program in the United States [abstract no. 0611]. sleep. 2019;42(supp11):A243. doi:10.1093/sleep/zsz067.609

53. Kollb-Sielecka M, Demolis P, Emmerich J, Markey G, Salmonson T, Haas M. The European Medicines Agency review of pitolisant for treatment of narcolepsy: summary of the scientific assessment by the committee for medicinal products for human use. Sleep Med. 2017;33:125-129. doi:10.1016/j.sleep.2017.01.002

54. Kallweit U, Triller A. Effects of pitolisant on nighttime sleep [abstract no. 0613]. sleep. 2019;42(suppl1):A244. doi:10.1093/ sleep/zsz067.611

55. Huyts B, Brabant C, Tirelli E. Pitolisant and intravenous cocaine self-administration in mice. Eur J Pharmacol. 2019;851:63-68. doi:10.1016/j.ejphar.2019.02.019

56. Lehert P, Falissard B. Multiple treatment comparison in narcolepsy: a network meta-analysis. Sleep. 2018;41.

57. Roth T, Dauvilliers Y, Guinta D, Alvarez-Horine S, Dynin E, Black J. Effect of sodium oxybate on disrupted nighttime sleep in patients with narcolepsy. J Sleep Res. 2017;26(4):407-414. doi:10.1111/ jsr. 12468

\section{Publish your work in this journal}

Nature and Science of Sleep is an international, peer-reviewed, open access journal covering all aspects of sleep science and sleep medicine, including the neurophysiology and functions of sleep, the genetics of sleep, sleep and society, biological rhythms, dreaming, sleep disorders and therapy, and strategies to optimize healthy sleep.
The manuscript management system is completely online and includes a very quick and fair peer-review system, which is all easy to use. Visit http://www.dovepress.com/testimonials.php to read real quotes from published authors. 